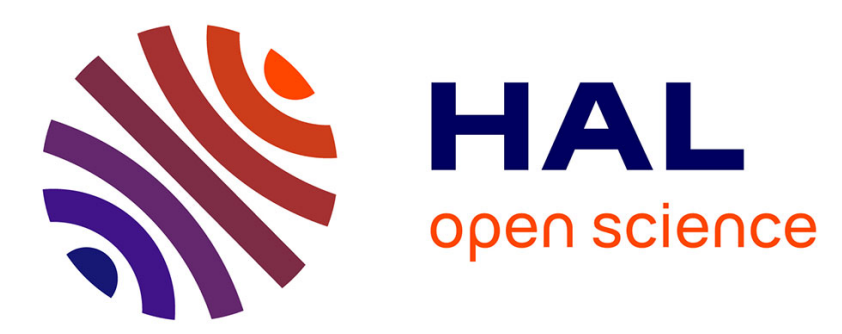

\title{
Surface nanoprocessing with nondiffracting femtosecond Bessel beams
}

\author{
F. Courvoisier, P.-A. Lacourt, M. Jacquot, M. K. Bhuyan, L. Furfaro, J.M. \\ Dudley
}

\section{- To cite this version:}

F. Courvoisier, P.-A. Lacourt, M. Jacquot, M. K. Bhuyan, L. Furfaro, et al.. Surface nanoprocessing with nondiffracting femtosecond Bessel beams. Optics Letters, 2009, 34 (20), pp.3163-3165. 10.1364/OL.34.003163 . hal-00453776

\section{HAL Id: hal-00453776 https://hal.science/hal-00453776}

Submitted on 5 May 2021

HAL is a multi-disciplinary open access archive for the deposit and dissemination of scientific research documents, whether they are published or not. The documents may come from teaching and research institutions in France or abroad, or from public or private research centers.
L'archive ouverte pluridisciplinaire $\mathbf{H A L}$, est destinée au dépôt et à la diffusion de documents scientifiques de niveau recherche, publiés ou non, émanant des établissements d'enseignement et de recherche français ou étrangers, des laboratoires publics ou privés. 


\title{
Surface nanoprocessing with nondiffracting femtosecond Bessel beams
}

\author{
F. Courvoisier,* P.-A. Lacourt, M. Jacquot, M. K. Bhuyan, L. Furfaro, and J. M. Dudley \\ Département d'Optique P. M. Duffieux, Institut FEMTO-ST, UMR CNRS 6174, Université de Franche-Comté, \\ 25030 Besançon, France \\ *Corresponding author: francois.courvoisier@femto-st.fr
}

\begin{abstract}
We demonstrate the application of nondiffracting Bessel beams for reproducible nanometric-scale feature patterning in glass. A femtosecond pulse zero-order Bessel beam with a central spot radius of $360 \mathrm{~nm}$ was used to write $500 \mathrm{~nm}$ radius nanocraters over a longitudinal positioning range exceeding $20 \mu \mathrm{m}$, with a variation in radius of less than $10 \%$. The use of Bessel beams significantly reduces constraints on critical sample positioning in the nanoscale writing regime, enabling the use of femtosecond pulses for fast inscription of nanometer-scale features over large sample areas.
\end{abstract}

Femtosecond laser ablation has developed into a versatile technique for the structural modification of a wide range of materials. The ultrafast light-matter interaction time leads to a deterministic ablation process and yields a high degree of machining precision and reproducibility. Although the physics of femtosecond ablation is complex, a characteristic feature is the presence of a precisely defined fluence threshold below which no ablation is observed. The threshold effect is significant in that it allows structuring over characteristic dimensions smaller than the focusing spot size diameter, even below the laser wavelength $[1,2]$.

Motivated by applications in producing and prototyping subwavelength structures for plasmonic and material applications, there has been much recent research toward extending femtosecond pulse machining technology into the nanoscale regime [3,4]. The use of femtosecond pulses for nanoscale writing, however, typically requires a strong focusing with high NAs. However, since the Rayleigh range is of the order of the spot radius, precise positioning of the material sample with respect to the incident beam waist then becomes critical, particularly with nearthreshold fluences and when only a few laser shots per site are required [5]. This critical positioning constraint imposes severe limits on sample flatness, which, for large samples $\left(\sim \mathrm{cm}^{2}\right)$, requires an active compensation of sample tilt and irregularity and complex setups based on high resolution confocal microscopy or optical trapping [6,7]. This additional complexity, however, significantly reduces the potential processing speed and acts as a bottleneck to the wider application of femtosecond machining technology in nanophotonics.

In this Letter, we describe the use of nondiffracting Bessel beams to overcome this problem. We extend previous technological applications of Bessel beams for index modification with 5-10 $\mu \mathrm{m}$ dimensions [8] into the important nanoscale regime, achieving nanometric scale surface structuring using femtosecond ablation with noncritical sample positioning exceeding the Rayleigh range by 1 order of magnitude. We explicitly characterize the longitudinal evolution of a $360 \mathrm{~nm}$ radius Bessel beam generated from $100 \mathrm{fs}$ pulses at $800 \mathrm{~nm}$ and demonstrate its application to write $500 \mathrm{~nm}$ radius nanocraters over a longitudinal positioning range exceeding $20 \mu \mathrm{m}$ with a variation in nanocrater radius of less than $10 \%$. The positioning range achieved with the Bessel beam represents 1 order of magnitude improvement relative to the conventional Gaussian beam focusing used to generate structures on the same scale and opens the way for the practical implementation of femtosecond machining to realize nanoscale photonic structures.

Bessel beams constitute a class of solutions to the Helmholtz equation with rotational symmetry. These beams have been primarily used for optical manipulation, for nonlinear optics, for their self-healing properties, [9] and for waveguide direct writing in glasses $[8,10]$. The amplitude of a zero-order Bessel beam can be described as [11]

$$
E(\rho, z)=e^{j \beta z} J_{0}\left(k_{t} \rho\right),
$$

where $k_{0}$ is the wave vector and $\rho$ is the radial coordinate. $\gamma$ and $k_{t}$ are defined as $k_{t}=k_{0} \sin (\gamma)=2 \pi / \rho_{0}$ and $\beta=k_{0} \cos (\gamma)$, with $\rho_{0}$ being the transverse period of the Bessel beam. $\gamma$ can be interpreted as the ray angle relative to the optical axis. Although ideal Bessel beams are invariant during propagation, the finite extension of an experimental Gaussian beam of waist $w_{0}$ leads to an on-axis intensity (from an initial point defined at $z=0$ ) that can be approximated by $[12,13]$

$$
I(z)=2 \pi I_{0} z \gamma^{2} \exp \left(-2 \gamma^{2} z^{2} / w_{0}^{2}\right) \quad \text { for } z>0 .
$$

To illustrate how Bessel beams reduce sample positioning constraints, the solid curve in Fig. 1 compares the expected evolution from Eq. (2) of the on-axis intensity for Bessel and Gaussian beams. The spot size used (360 $\mathrm{nm}$ radius) is chosen to match our experimental parameters below. Approximating the size of the single shot ablation damage site to the beam diameter, where the local intensity exceeds the ablation threshold [14], the longitudinal range $\Delta z$ over 


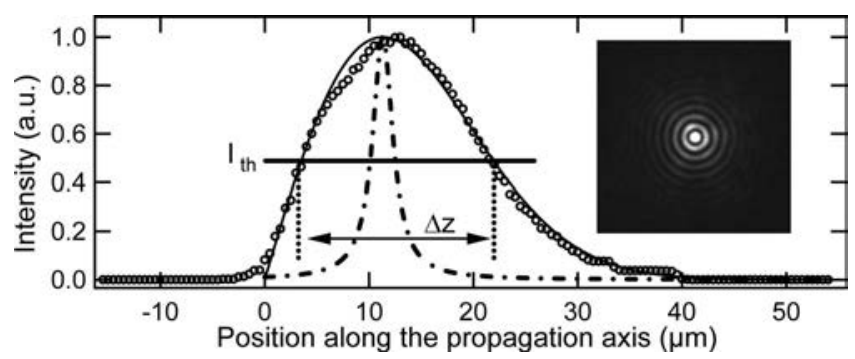

Fig. 1. Comparison of the on-axis intensity evolution along the propagation direction for Gaussian (dasheddotted curve) and Bessel [solid curve, from Eq. (2)] beams with an identical spot radius of $360 \mathrm{~nm}$. The experimental values are shown as open circles. Inset, image of the experimental beam. The central spot radius is $360 \mathrm{~nm}$ at half-maximum.

which near-uniform nanodamage is produced can be estimated as the distance along the beam axis where the on-axis intensity exceeds this threshold. For the incident fluence, $F=2 F_{\text {th }}$, this range is $\Delta z_{B}=18 \mu \mathrm{m}$ for our experimental parameters, whereas the range falls to $\Delta z_{G}=2.35 \mu \mathrm{m}$ for a Gaussian beam of the same spot size. Maintaining the sample position within this range for a Gaussian beam is clearly technically difficult during patterning over large samples. In contrast, the Bessel beam has an effective patterning volume with an aspect ratio of 25 and allows ablation over a positioning range compatible with common values of total thickness variation (TTV) of commercial wafers (typically $<10 \mu \mathrm{m}$ ).

The potential of Bessel beams for noncritical sample positioning in laser ablation was confirmed using the setup shown in Fig. 2 to produce a Bessel beam of submicrometer spot size while maintaining imaging capabilities. The femtosecond Bessel beam was produced by a spatial light modulator (SLM) combined with a telescope arrangement, whose second lens is the imaging microscope objective (MO). A specific feature of this setup is that it allows the generation of longitudinally extended Bessel beams of submicrometer dimension while simultaneously preserving the long working distance of the MO (typically $3 \mathrm{~mm}$ ).

The SLM imprints a phase pattern in a similar way to an axicon, i.e., of the form $\phi=2 \pi \rho / \rho_{0}$. With the SLM phase modulation lying in the interval of $0-2 \pi$ instead of $-\pi$ to $\pi$ for an axicon or a grating, the zeroth order does not contain the spatial modulation information, and the Bessel beam is instead diffracted into first order. To eliminate the other orders, we therefore added to the phase modulation the phase of a plane wave with a tilt of $5 \mathrm{mrad}$, allowing spatial

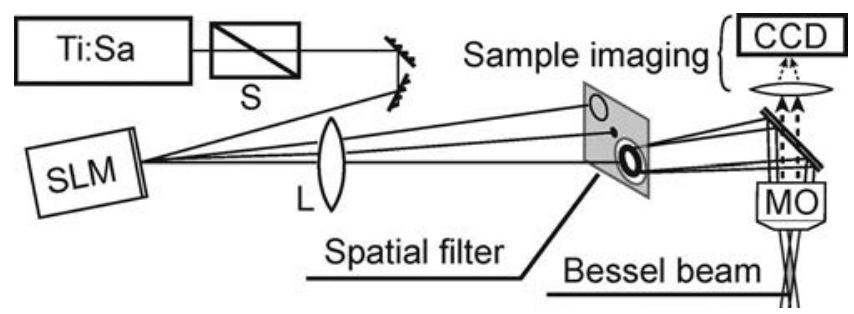

Fig. 2. Experimental setup: SLM, spatial light modulator; $\mathrm{S}$, optical shutter; MO, microscope objective; L, lens. separation and filtering of the desired +1 order with an iris.

The amplified laser source emits $100 \mathrm{fs}$ laser pulses at $800 \mathrm{~nm}$. An independent Pockels cell system with a thin film polarizer acts as an optical shutter allowing single shot illumination. The pulse energy is varied using neutral density filters. The SLM (Hamamatsu PAL-SLM) is nonpixelated thanks to optical addressing, with a $2 \mathrm{~cm}$ active window. An $f$ $=1 \mathrm{~m}$ focal distance lens is combined with a longworking-distance material science MO to form a telescope with a magnification of $M=1 / 278$. The infinitycorrected objective has a magnification of $50 \times$ and a plan-Fluor correction for NA=0.8. The spatial filtering of the spurious diffraction modes is performed in the focal plane of the lens. The produced zero-order Bessel beam was characterized by imaging the beam profiles onto a CCD camera for different positions along the beam propagation with a step size of $0.5 \mu \mathrm{m}$. A $40 \times \mathrm{MO}$ was mounted on a motorized translation stage, and the focal plane was conjugated with the CCD camera by a lens with a focal length of $f=20 \mathrm{~cm}$. The imaging magnification was $40 \times$.

The inset of Fig. 1 shows a CCD camera image of the beam profile with $\rho_{0}=1.8 \mu \mathrm{m}$, corresponding to a central spot radius of $360 \mathrm{~nm}$ at half-maximum. The experimental evolution of the central lobe intensity $I(r=0, z)$ is plotted against the propagation distance in Fig. 1 (open circles) and is clearly in very good agreement with that expected from Eq. (2) [12].

To study noncritical $z$ positioning when nanomachining with Bessel beams, we compared the damage spots induced by single shot femtosecond laser ablation for different $z$ positions along the beam. Specifically, the front side of a borosilicate glass sample (Corning 0211) was illuminated by individual shots of the Bessel beam and displaced between each laser shot by $1 \mu \mathrm{m}$ steps along the beam direction and $10 \mu \mathrm{m}$ transversally. The sample orthogonality with respect to the beam propagation axis was ensured to be better than $1 \mathrm{mrad}$. After metallization, the glass sample was imaged with high resolution scanning electron beam microscopy (SEM) and the crater radii were determined by averaging measurements on SEM images performed on two perpendicular directions.

Figure 3 shows the evolution of the radii of holes drilled by single laser shots as a function of the distance along the beam axis, with peak fluence at twice the ablation threshold. At both extremities of the data series, where the local intensity just reaches the damage threshold, the ablated material is not fully removed, and the laser pulse creates only surface features (bumps). The radii of the bumps are plotted on the same figure. Between these extremities, where the local intensity exceeds the threshold, the damage consists of a crater with a radius of $550 \mathrm{~nm}$ and a central nanochannel with a radius of $50 \mathrm{~nm}$. The damage remains visually identical independent of longitudinal position over a distance of $\Delta z=20 \mu \mathrm{m}$. This range agrees well with the $18 \mu \mathrm{m}$ measured depth of focus of the Bessel beam. Over this range, the crater radius is near constant at $550 \mathrm{~nm} \pm 9.6 \%$. 


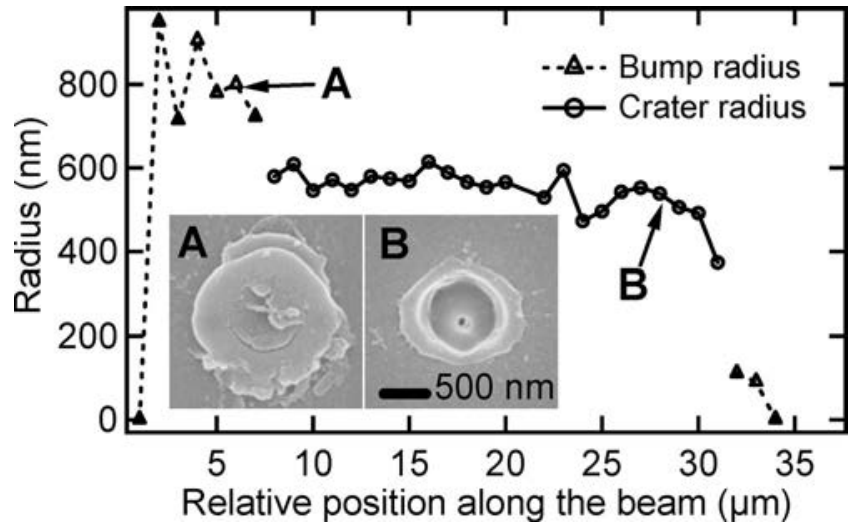

Fig. 3. Dependence of the mean crater radius drilled by single shot femtosecond laser pulses on a distance along the propagation direction of the Bessel beam. Insets correspond to SEM images at data points A and B. The morphology of craters over the position range of $10-30 \mu \mathrm{m}$ is near identical to image B.

In addition to these surface images, additional transverse characterization using focused ion beam milling and SEM imaging confirms the reproducibility of the nanocrater structure, allowing us to estimate the depths of the crater and nanohole structures as 500 $\mathrm{nm}$ and $1 \mu \mathrm{m}$, respectively. Moreover, at fluences far higher than the threshold, this approach represents a significant extension to alternative techniques that have demonstrated such high aspect ratio nanochannels with an elongated focal zone owing to nonlinear propagation and spherical aberration $[15,16]$.

The useful nanomachining range $\Delta z$ above is larger than typical wafer TTV values, enabling a sample tilt up to $2 \mathrm{mrad}$ for patterning over $1 \mathrm{~cm}$ while maintaining constant laser damage. This demonstrates that Bessel beams render sample positioning far less critical than the usual Gaussian focusing, without compromising the size of the laser induced damage. The fact that the longitudinal extent of the produced "micro-Bessel beam" is much smaller than depths of focus generally reported $[9,11]$ arises from the use of a telescope to reduce the beam diameter, thus also reducing the depth of focus by the square of the magnification. Microaxicons could give similar depths of focus for an identical central lobe diameter, but do not offer the same flexibility as our setup for online imaging and variation in the focusing conditions, and would excessively reduce the working distance. The only requirement imposed by the use of Bessel beams is that the fluence needs to stay below the limit of three times the ablation threshold to avoid machining by the lateral lobes. However, this is not a practical constraint as femtosecond nanopatterning is performed near the threshold, far below this limit. Additional improvements may be obtained by trun- cating the Bessel-Gauss beams since this allows for the removal of peripheral lobes (needle beams) [17].

In conclusion, we have demonstrated that nondiffracting Bessel beams can be successfully used to overcome critical sample positioning for ultrafast laser surface nanopatterning. A femtosecond zero-order Bessel beam was generated with a central spot radius of $360 \mathrm{~nm}$. Identical damage spots in fused silica have been demonstrated over a longitudinal distance of $20 \mu \mathrm{m}$ with a variation of less than $10 \%$ of the crater radius. We anticipate that this approach will lead to a more widespread application of fast laser processing for patterning nanometric-scale surface features over large samples in nanophotonics.

The authors acknowledge the Conseil Régional de Franche-Comté, the Institut Universitaire de France, and the French Institut Carnot Programme for funding.

\section{References}

1. A. P. Joglekar, H. Liu, G. J. Spooner, E. Meyhofer, G. Mourou, and A. J. Hunt, Appl. Phys. B 77, 25 (2003).

2. R. R. Gattass and E. Mazur, Nat. Photonics 2, 219 (2008).

3. C. Reinhardt, R. Kiyan, S. Passinger, A. L. Stepanov, A. Ostendorf, and B. N. Chichkov, Appl. Phys. A 89, 321 (2007).

4. G. Della Valle, R. Osellame, and P. Laporta, J. Opt. A 11, 013001 (2009).

5. D. J. Hwang, C. P. Grigoropoulos, and T. Y. Choi, J. Appl. Phys. 99, 083101 (2006).

6. E. Vanagas, I. Kudryashov, D. Tuzhilin, S. Juodkazis, S. Matsuo, and H. Misawa, Appl. Phys. Lett. 82, 2901 (2003).

7. E. McLeod and C. Arnold, Opt. Express 17, 3640 (2009).

8. V. Zambon, N. McCarthy, and M. Piché, Proc. SPIE 7099, 70992J (2008).

9. D. McGloin and K. Dholakia, Contemp. Phys. 46, 15 (2005).

10. A. Marcinkevičius, S. Juodkazis, S. Matsuo, V. Mizeikis, and H. Misawa, Jpn. J. Appl. Phys. Part 2 40, L1197 (2001).

11. J. Durnin, J. Miceli, and J. H. Eberly, Phys. Rev. Lett. 58, 1499 (1987).

12. V. Jarutis, R. Paskauskas, and A. Stabinis, Opt. Commun. 184, 105 (2000)

13. J. Amako, D. Sawaki, and E. Fujii, J. Opt. Soc. Am. B 20, 2562 (2003).

14. J. Koch, F. Korte, C. Fallnich, A. Ostendorf, and B. N. Chichkov, Opt. Eng. 44, 051103 (2005).

15. Y. V. White, X. Li, Z. Sikorski, L. M. Davis, and W. Hofmeister, Opt. Express 16, 14411 (2008).

16. S. I. Kudryashov, G. Mourou, A. Joglekar, J. F. Herbstman, and A. J. Hunt, Appl. Phys. Lett. 91, 141111 (2007).

17. R. Grunwald, M. Bock, V. Kebbel, S. Huferath, U. Neumann, G. Steinmeyer, G. Stibenz, J.-L. Néron, and M. Piché, Opt. Express 16, 1077 (2008). 